\title{
In-focus Cryo-EM Images Using a Hybrid Single-sideband/Double-sideband Aperture
}

\author{
Robert M. Glaeser ${ }^{1},{\text { Bong-Gyoon } \text { Han }^{1} \text {, Simone Sassolini }}^{2},{\text { Jian } \mathrm{Jin}^{2} \text {, and Stefano Cabrini }}^{3}$ \\ 1. Life Sciences Division, LBNL, University of California, Berkeley, CA, USA \\ 2. Engineering Division, LBNL, University of California, Berkeley, CA, USA \\ 3. Molecular Foundry and Materials Sciences Division, LBNL, University of California, Berkeley, CA, \\ USA
}

A microfabricated "phase-contrast" aperture has been described previously by Buijsse et al. [1], which incorporates an electron-opaque half-circle in the center (supported by a narrow beam). This type of aperture, referred to as a tulip aperture for short, is designed to provide high-contrast images of cryo-EM specimens at Scherzer defocus. The opaque half circle produces single-sideband contrast at low spatial frequencies, where the contrast transfer for double-sideband images is very poor, while retaining doublesideband contrast over most of the remaining spatial frequencies. While the contrast transfer within the single-sideband region is 0.5 , independent of defocus, a systematic phase shift of 90 degrees, together with any wave-aberration due to defocus - or due to charging of the aperture, shows up in the phase of the computed Fourier transform of the image. At Scherzer defocus the phase error due to defocus remains small, but the phase error due to charging could be large. We now report results of experiments in which images of monolayer crystals of streptavidin were used to characterize the performance achievable with this type of aperture. The signal in the computed Fourier transform of these images is confined to diffraction spots, making it relatively easy to determine whether charging of the aperture has introduced significant phase error. In addition, the signal-to-noise ratio (SNR) of these diffraction spots provides an easy way to judge the significance of the data as a function of resolution.

To evaluate the extent to which charging introduced phase errors, two successive images of the same area of the specimen were recorded. The first image was recorded at Scherzer defocus with the tulip aperture, and the second was recorded at $\sim 3.7$ micrometer underfocus, without the aperture. As is shown in Figure 1, the phases corresponding to the single-sideband portion of the Fourier transform of the image are very similar, implying that the tulip aperture was virtually free of charging. The space-group symmetry of the streptavidin crystal requires the phases to be zero or 180 degrees for untilted specimens. We believe that different areas of the specimen are often tilted by as much as 5 degrees, thus allowing some phases to deviate from these values. We also believe that this tilt angle changes by a few degrees while the images are being recorded, thus causing some discrepancy in values for the first and second image respectively. As evidence that specimen tilt angle changes we show an example in which the intensity of adjacent diffraction spots increased in the second image, when the intensity of most spots decreased, as expected due to radiation damage. Figure 2 illustrates the high contrast that the tulip aperture provides for in-focus images, even when the particle size is very small. Individual streptavidin monomers, $\mathrm{MW}=55 \mathrm{kDa}$, are relatively easy to see, especially in images produced after correction of the systematic phase error of 90 degrees. Finally, when data are merged from several crystals, it is clear that the resolution achievable with the tulip aperture is in the range of 3 to 4 Angstroms.

The working lifetime of the tulip apertures is currently limited to just a few days. We thus hope to make essentially disposable apertures by using lithography for fabrication, rather than a FIB (as at present). In addition, we continue to do further experiments to increase the working lifetime [2]. 


\section{References:}

[1] B. Buijsse et al., Ultramicroscopy 111 (2011), 1688.

[2] The authors acknowledge funding from NIH grant GM083039, and the support of the Molecular Foundry, a DOE-supported user facility at LBNL.
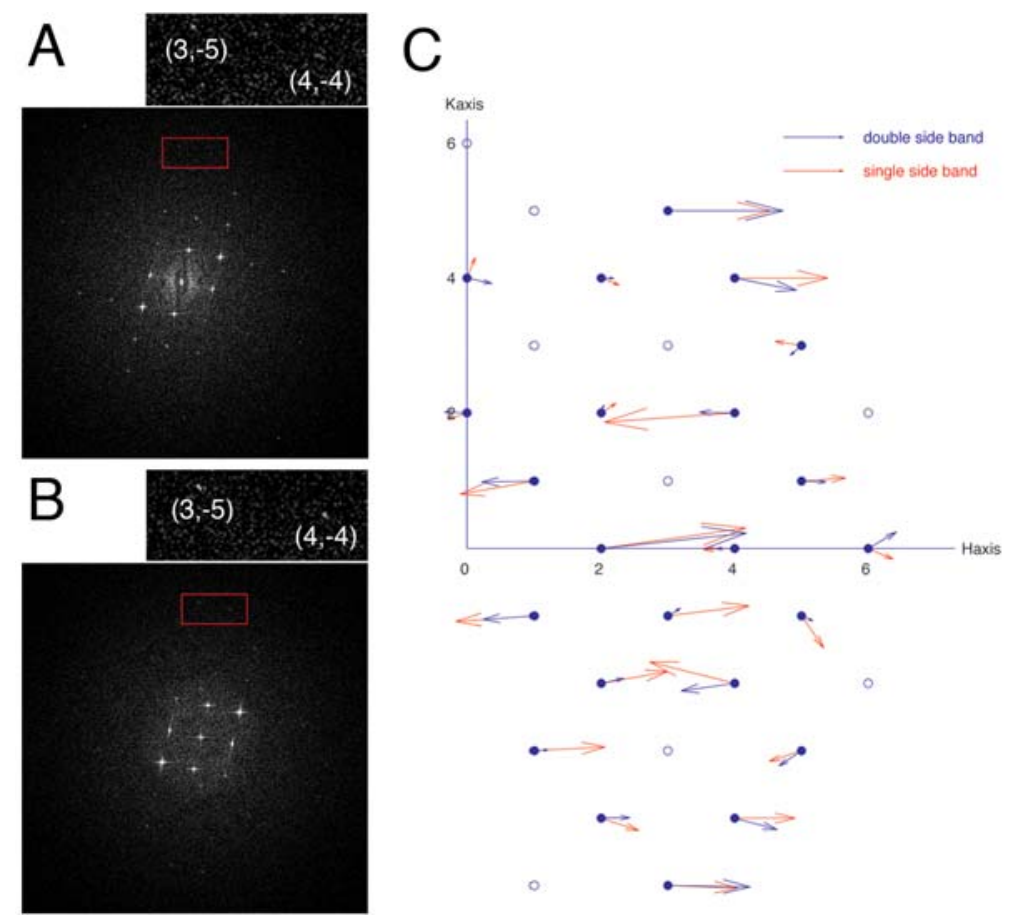

Figure 1. Comparison of the Fourier transforms of images of a streptavidin monolayer crystal recorded with or without the tulip aperture, respectively. An electron exposure of $\sim 1500$ electrons $/ \mathrm{nm}^{2}$ was used in each case. (A) The Fourier transform of the first image, with the insert showing an enlargement of the boxed area. (B) The Fourier transform of the second image, with the insert again showing an enlargement of the boxed area. (C) Graphical representation of the structure-factor phases. The similar direction of vectors shows that there does not appear to be a systematic phase error in the single-sideband portion of the Fourier spectrum.

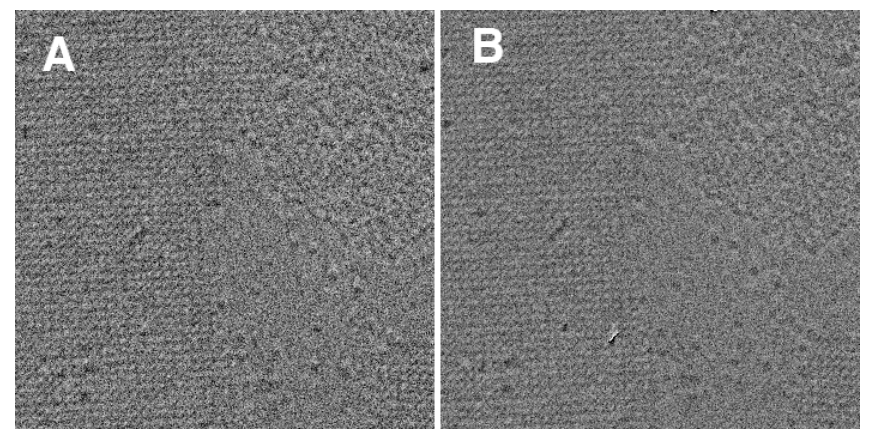

Figure 2. (above) Cryo-EM images of the streptavidin sample (A) before and (B) after correction of the systematic, 90-degree phase shift inherent in single-sideband images.

Figure 3 (right). Plot of the values of signalto-noise ratio for diffraction spots in data merged from several images. Spots given an IQ (image quality) score between 1 and 4 are highly significant.

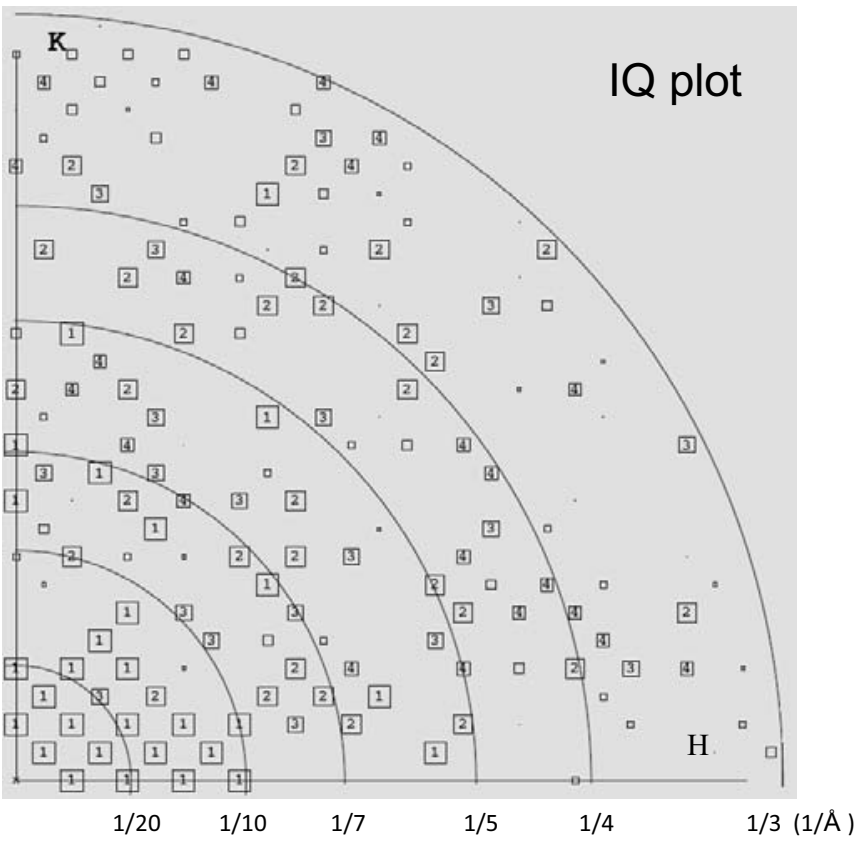

